\section{Risk factors for the development of lithium-induced}

\section{polyuria}

\author{
K. L. L. MOVIG, R. BAUMGARTEN, H. G. M. LEUFKENS, \\ J. H. M. VAN LAARHOVEN and A. C. G. EGBERTS
}

Lithium salts have been in widespread use since the 1960s for the treatment of several psychiatric disorders, including bipolar disorders and depression (MullerOerlinghausen et al, 2002). Since the introduction of lithium therapy a variety of studies have investigated its side-effects, such as cutaneous reactions, thyroid disorders and urine-concentration abnormalities. Polyuria has been estimated to occur in $15-40 \%$ of all patients treated with lithium. Clinically established nephrogenic diabetes insipidus has been found in about $12 \%$ of all patients treated with lithium (Boton et al, 1987).

Early studies found that 24-h urine volumes were increased and urinary osmolality was decreased in patients using lithium concurrently with psychotropic medication (Bone et al, 1980; Bendz et al, 1983). However, these studies were conducted before the introduction of many new psychotropic drugs, especially the second-generation antidepressants. More recently, it has been found that patients treated with both lithium and unspecified psychotropic drugs had a lower urinary concentrating capacity and glomerular filtration rate than patients taking lithium alone (Bendz et al, 1994).

The objectives of our study were to estimate the prevalence of polyuria associated with the use of lithium and to identify additional risk factors, especially concurrently used medication, that predispose patients on lithium to develop polyuria.

\section{METHOD}

\section{Setting and study population}

A prospective follow-up study was designed to assess the prevalence and determinants of polyuria among lithium users. The source population of our study was all psychiatric patients with a diagnosis of affective disorder attending the lithium clinic of a psychiatry department within the period September 2001 to January 2002. All patients using lithium for at least 1 month were asked to participate in this study during their routine visits (scheduled once every 3 months) to the out-patient lithium clinic in the psychiatry department. All data were anonymously processed and all participants gave written informed consent for inclusion in this study.

\section{Outcome}

The primary end-point of this study was the presence of polyuria, defined quantitively as a daily (24-h) urine volume greater than 3 litres (Baylis, 1996). Patients were given clear instructions (both verbal and written) regarding the collection of the urine samples and were encouraged to contact us when there were any questions. The volume of the urine was determined and creatinine concentrations and osmolality were measured by standard laboratory methods. If the urinary creatinine content was less than $6.0 \mathrm{mmol}$ per day, the $24-\mathrm{h}$ urine collection was considered inaccurate. Fluid intake was estimated by means of a written questionnaire, asking patients to report fluid consumption during the $24-\mathrm{h}$ urine sampling period.

\section{Determinants}

Serum lithium concentrations were measured in the routine laboratory therapeutic drug monitoring programme. The cumulative amount of ingested lithium was expressed as the product of time on lithium and the average daily dosage, determined from medical records. Patients were defined as current drug users if the prescription lasted until the day of the visit to the lithium clinic. A psychiatrist or nurse practitioner obtained drug prescription data during the patient's visit, and the community pharmacy was consulted. Data on comorbidity and smoking behaviour were obtained from medical records.

Antidepressant drugs were classified into two groups. The first group consisted of antidepressants predominantly acting on the serotonergic system (serotonergic antidepressants), which included clomipramine, fluoxetine, paroxetine, sertraline, trazodone and venlafaxine. The second group consisted of antidepressants that have less potency to inhibit the serotonin reuptake mechanism, including amitriptyline, imipramine, maprotiline and nortriptyline. Clomipramine, trazodone 
and venlafaxine were included in the first group because these compounds are known to be potent antagonists of the serotonin reuptake mechanism (Tatsumi et al, 1997).

\section{Data analysis}

For all patients the prevalence of each characteristic on the inclusion date was determined. An analysis of variance (Student's $t$-test) was used to compare frequencies of continuous variables for participants with and without polyuria. Differences in proportions of categorical variables were assessed for significance by a chi-squared test.

To estimate a possible association between potential risk factors and polyuria, crude and adjusted odds ratios were calculated as measures of relative risk using logistic regression and were presented with a $95 \%$ confidence interval. The final logistic regression model included age and gender and all univariately associated (at $P \leqslant 0.1)$ risk factors for polyuria. In order to study and adjust for potential confounding factors, additional data on concomitant medication (antidepressants, diuretics, antihypertensives, neuroleptics) and comorbidity (diabetes mellitus, hypertension, thyroid disease) were collected. All calculations were carried out using the Statistical Package for the Social Sciences, version 10.0.

\section{RESULTS}

The final study population consisted of 75 patients. All these patients gave informed consent and collected adequate $24-\mathrm{h}$ urine samples. Six patients were excluded for various reasons. Table 1 summarises the characteristics of the patients, stratified according to presence or absence of polyuria. The mean age of the patients was 52 years; the women were slightly older (54 years $v .49$ years) but this was not statistically significant $(P=0.069)$. In the group as a whole, 46 patients $(61 \%)$ were female and the average lithium treatment duration was 4.7 years. For 44 patients $(59 \%)$ the primary psychiatric indication for lithium was bipolar affective disorder and for 31 patients $(41 \%)$ it was major depressive disorder. There was no diagnosis of schizoaffective disorder. In the group with major depressive disorder, lithium was always added to an antidepressant as an augmentation strategy. In the group of patients with bipolar (I and II) disorder an antidepressant was added when lithium alone

Table I Baseline demographic and medical characteristics of the study participants

\begin{tabular}{|c|c|c|c|}
\hline & $\begin{array}{l}\text { All lithium users } \\
\qquad(n=75)\end{array}$ & $\begin{array}{l}\text { With polyuria } \\
\qquad(n=28)\end{array}$ & $\begin{array}{l}\text { Without polyuria } \\
\qquad(n=47)\end{array}$ \\
\hline \multicolumn{4}{|l|}{ Demographic characteristics } \\
\hline Age (years): mean (s.d.) & $52(12)$ & $51(13)$ & $54(9)$ \\
\hline \multicolumn{4}{|l|}{ Gender: $n(\%)$} \\
\hline Male & $29(39)$ & II (39) & $18(38)$ \\
\hline Female & $46(61)$ & $17(61)$ & $29(62)$ \\
\hline \multicolumn{4}{|l|}{ Diagnosis: $n(\%)$} \\
\hline Major depressive disorder & $3 I(4 I)$ & $9(32)$ & $22(47)$ \\
\hline Bipolar I & $38(5 \mathrm{I})$ & $16(57)$ & $22(47)$ \\
\hline Bipolar II & $6(8)$ & $3(I I)$ & $3(6)$ \\
\hline \multicolumn{4}{|l|}{ Lithium use (years) } \\
\hline Mean (range) & $4.7(0.4-30.7)$ & $6.0(0.6-30.7)$ & $3.9(0.4-14.6)$ \\
\hline$<3$ years: $n(\%)$ & $35(47)$ & $10(36)$ & $25(53)$ \\
\hline$\geqslant 3$ years: $n(\%)$ & $40(53)$ & $18(64)$ & $22(47)$ \\
\hline Dosage (mg'): mean (range) & $919(400-1800)$ & $958(400-1800)$ & $895(400-1400)$ \\
\hline Lithium dosing once daily: $\boldsymbol{n}(\%)$ & $53(7 \mathrm{I})$ & $19(68)$ & $34(72)$ \\
\hline Serum lithium (mmol/l): mean (s.d.) & $0.78(0.17)$ & $0.81(0.15)$ & $0.76(0.18)$ \\
\hline BMI $\left(\mathrm{kg} / \mathrm{m}^{2}\right):$ mean (s.d.) & $26.1(4.2)$ & $25.9(4.3)$ & $26.2(4.2)$ \\
\hline Smoking & $23(3 I)$ & $10(36)$ & $13(28)$ \\
\hline \multicolumn{4}{|l|}{ Drug use and comorbidity } \\
\hline \multicolumn{4}{|l|}{ Antidepressants: $n$ (\%) } \\
\hline Serotonergic & $25(33)$ & $14(50)$ & II (23) \\
\hline Other & $16(21)$ & $4(14)$ & $12(26)$ \\
\hline Antipsychotics: $n(\%)$ & $6(8)$ & $0(0)$ & $6(13)$ \\
\hline Benzodiazepines: $\boldsymbol{n}$ (\%) & $15(20)$ & $6(21)$ & $9(19)$ \\
\hline Diuretics: $\boldsymbol{n}(\%)$ & $0(0)$ & $0(0)$ & $0(0)$ \\
\hline Diabetes mellitus: $\boldsymbol{n}(\%)$ & $2(3)$ & $0(0)$ & $2(4)$ \\
\hline Hypertension: $\boldsymbol{n}(\%)$ & $9(12)$ & $3(I I)$ & $6(13)$ \\
\hline Thyroid disorder: $n$ (\%) & $16(21)$ & $3(I I)$ & $13(28)$ \\
\hline
\end{tabular}

BMI, body mass index.

I. Lithium carbonate.

did not prevent depression. The lithium serum concentrations were not statistically different between the two patient groups $(P=0.258)$. Twenty-eight patients $(37 \%)$ were identified as having polyuria. Outcome parameters for patients with and without polyuria are presented in Table 2 . There was no difference between the two groups in 24-h creatinine excretion. In both patient groups, the intake volume equalled the 24-h urine output volume. Half of the patients with polyuria were current users of serotonergic antidepressants, whereas less than a quarter of the non-polyuria patients $(23 \%)$ were current users of these drugs. Users of serotonergic antidepressants had a three times higher risk (unadjusted relative risk 3.06, 95\% CI 1.04-9.00) for polyuria compared with non-recipients of these agents (Table 3). Concurrent use of benzodiazepines, hypertension, lithium dosing frequency and smoking were not significantly associated with polyuria. Thyroid disorders were inversely associated with polyuria, but this was not statistically significant.

After adjustment for potential confounding factors, the clear association between the use of serotonergic antidepressants and polyuria in this cohort of lithium users remains: adjusted relative risk 4.25 (95\% CI 1.15-15.68) compared with nonrecipients. The most frequently used serotonergic antidepressants were clomipramine and paroxetine. Stratified analysis showed that patients using lithium for 3 years or more were at a higher risk $(\mathrm{OR}=$ 2.65 , 95\% CI $0.87-8.07$ ) of polyuria 
of polyuria (Bowen et al, 1991). However, researchers are not unanimous. O’Donovan et al (1993) found that switching to a single daily dose of lithium did not reduce the 24-h urine volume. In our study urine volume was not significantly related to the dosing regimen. Both thiazides and potassium-sparing diuretics have been shown to ameliorate lithium-induced polyuria (Martin, 1993). Diuretics were not used in our patient population.

Smoking impairs urine excretion by increasing endogenous arginine vasopressin (AVP) secretion (Allon et al, 1990). However, in our study smoking was not found to be associated with a decreased urinary volume. Patients with thyroid disorder seemingly had a lower risk of polyuria. No possible pharmacological explanation was found in the research literature.

\section{Pharmacological explanation}

The mechanism of lithium-induced polyuria is biologically explicable. Under normal physiological circumstances AVP binds to vasopressin type 2 receptors located in the basolateral membrane of the renal collecting-duct cells. This binding initiates a cascade, triggering the insertion of vesicles containing the aquaporin-2 water channel into the normally watertight apical membrane, to cause increased water permeability followed by water resorption, leading to concentrated urine production (Deen \& Knoers, 1998). In rat models lithium appeared to cause polyuria through downregulation of aquaporin-2 expression (Marples et al, 1995), which has been confirmed in humans in healthy individuals under lithium treatment (Baumgarten et $a l, 2000)$. It has been suggested that lithium acts by inhibiting adenylate cyclase activity in the collecting-duct cells, preventing the production of cyclic adenosine monophosphate (cAMP), the second messenger for vasopressin.

A pharmacological explanation for serotonergically induced polyuria has not been explored. So far, it has been accepted that antidepressants are effective through interaction with certain key receptors in serotonergic, noradrenergic and/or dopaminergic neurotransmission systems (Stahl, 1998). It is thought that lithium enhances antidepressant efficacy by increasing serotonergic transmission. The value of lithium augmentation has been demonstrated with a wide range of antidepressants, including serotonergic agents (Zullino \& Baumann, found in this study is in line with other published values, although reported rates of lithium-associated polyuria vary widely is lacking. Our findings are similar to those patients treat (1994), who reported chotropic agents had a decreased urina concentration capacity and increased 24the psychotropic agents were not asses separately and no relative risk estimate

High serum levels of lithium have been reportedly associated with significantly more cases of polyuria than have low serum levels (Maj et al, 1986). However, our study found no statistically significant relationship between serum lithium levels and polyuria. The small number of cases might be a reason for this observation. It has been suggested that lithium administration in a single daily dose rather than multiple daily doses might accelerate the tubular regeneration process and decrease the risk 
2001). No clinically relevant pharmacokinetic interaction has been found between lithium and serotonin reuptake inhibitors (Mitchell, 1997). Double-blind trials have not demonstrated that adverse effects occurred frequently using the combination of lithium and serotonergic antidepressants (Fava et al, 1994); however, these trials were not designed to estimate the prevalence of such adverse effects, including polyuria.

Serotonergic antidepressants are associated with urinary problems in daily clinical practice. It has been recorded that serotonergic drugs can induce hyponatraemia (Movig et al, 2002), supposedly by inappropriate secretion of antidiuretic hormone, which eventually leads to a decrease in urine production. Since we found a statistically significant additional effect of serotonergic antidepressants on lithium-induced polyuria, it is likely that the effect of serotonergic medication on lithium-induced polyuria is explained by a drug interaction at the tubular level.

\section{Limitations of the study design}

Our study has some potential limitations. Selection bias is a potential threat to study validity and occurs whenever the inclusion of patients is in some way associated with the outcome of interest. However, the psychiatrists were not informed about the primary objective of this study; they only recruited the patients and instructed them in the urine collection protocol. It is therefore unlikely that users of serotonergic agents were more frequently included than patients using other (or no) antidepressants. A second concern is the possibility of observer bias. This was minimised by medical record review after the patient had visited the lithium clinic to verify the study forms filled in by the psychiatrist.

Although the study was sufficiently powerful to detect a number of risk factors, it was somewhat limited by its small size. The wide confidence intervals around some of the odds ratios are a reflection of this limitation.

A dichotomous cut-off point of 3 litres of urine per day for polyuria has been used in the logistic regression model. Such a cutoff level is widely accepted for research purposes (Baylis, 1996), but it still might be arbitrary. Therefore, we also performed analyses using continuous measures of 24-h urine output as the dependent variable, i.e. linear regression analysis. We

\section{CLINICAL IMPLICATIONS}

Polyuria is a well-known and frequently occurring side-effect of lithium therapy.

- Physicians should be aware of the increased risk of lithium-induced polyuria in patients concomitantly using serotonergic antidepressants.

These findings are in themselves insufficient reason to discontinue treatment.

\section{LIMITATIONS}

The number of patients in this study was small.

Selection bias might have occurred, albeit minimally.

Generalisability may be limited because recruitment was restricted to a single clinic.

K. L. L. MOVIG, PhD, Hospital Pharmacy Midden-Brabant, Tilburg, and Utrecht Institute for Pharmaceutical Sciences, Utrecht; R. BAUMGARTEN, MD, PhD, Atrium Medical Centre, Heerlen; H. G. M. LEUFKENS, PhD, Utrecht Institute for Pharmaceutical Sciences, Utrecht; J. H. M. VAN LAARHOVEN, MD, St Elisabeth Hospital, Tilburg; A. C. G. EGBERTS, PhD, Hospital Pharmacy Midden-Brabant, Tilburg, and Utrecht Institute for Pharmaceutical Sciences, Utrecht

Correspondence: Professor dr Antoine Egberts, Department of Pharmacoepidemiology and Pharmacotherapy, Utrecht Institute for Pharmaceutical Sciences, PO Box 80082, 3508 TB Utrecht, The Netherlands.Tel: 30253 7324; fax: 30253 9166; e-mail: A.C.G.Egberts@pharm.uu.n

(First received 25 April 2002, final revision 18 October 2002, accepted 30 October 2002)

found that patients who had been treated with selective serotonin reuptake inhibitors (SSRIs) and lithium salts concurrently had significantly greater urinary output (mean difference $941 \mathrm{ml}, 95 \%$ CI $280-1601$; $P=0.006$ ) than patients treated with lithium only. The logistic regression model provided a comparable statistically significant $P$ value, and in essence the same result, for the association between polyuria and treatment with both lithium and SSRIs compared with lithium use only.

\section{REFERENCES}

Allon, M., Allen, H., Deck, L., et al (1990) Role of cigarette use in hyponatremia in schizophrenic patients. American Journal of Psychiatry, 147, 1075-1077.

Baumgarten, R., van de Pol, M. H., Deen, P. M., et al (2000) Dissociation between urine osmolality and urinary excretion of aquaporin-2 in healthy volunteers. Nephrology, Dialysis, Transplantation, 15, II55-1161.

Baylis, P. H. (1996) Water and sodium homeostasis and their disorders. In Oxford Textbook of Medicine (eds D. J.Weatherall, J. G. G. Ledingham \& D. A. Warrel), pp. 3116-3126. Oxford: Oxford University Press.

Bendz, H., Andersch, S. \& Aurell, M. (1983) Kidney function in an unselected lithium population.
A cross-sectional study. Acta Psychiatrica Scandinavica, 68, 325-334.

_, Aurell, M., Balldin, J., et al (1994) Kidney damage in long-term lithium patients: a cross-sectional study of patients with 15 years or more on lithium. Nephrology, Dialysis, Transplantation, 9, 1250-1254.

Bone, S., Roose, S. P., Dunner, D. L., et al (1980) Incidence of side effects in patients on long-term lithium therapy. American Journal of Psychiatry, 137, 103-104.

Boton, R., Gaviria, M. \& Battle, D. C. (1987)

Prevalence, pathogenesis, and treatment of renal dysfunction associated with chronic lithium therapy. American Journal of Kidney Disease, 10, 329-345.

Bowen, R. C., Grof, P. \& Grof, E. (1991) Less frequent lithium administration and lower urine volume. American Journal of Psychiatry, 148, 189-192.

Deen, P. M. \& Knoers, N. V. (1998) Physiology and pathophysiology of the aquaporin-2 water channel. Current Opinion in Nephrology and Hypertension, $\mathbf{7}$. 37-42.

Fava, M., Rosenbaum, J. F., McGrath, P. J., et al (1994) Lithium and tricyclic augmentation of fluoxetine treatment for resistant major depression: a doubleblind, controlled study. American Journal of Psychiatry, 15I, 1372-1374.

Maj, M., Starace, F., Nolfe, G., et al (1986) Minimum plasma lithium levels required for effective prophylaxis in DSM III bipolar disorder: a prospective study. Pharmacopsychiatry, $19,420-423$.

Marples, D., Christensen, S., Christensen, E. I., et al (1995) Lithium-induced downregulation of aquaporin-2 
water channel expression in rat kidney medulla. Journal of Clinical Investigation, 95, 1838-1845.

Martin, A. (1993) Clinical management of lithiuminduced polyuria. Hospital and Community Psychiatry, 44, $427-428$.

Mitchell, P. B. (1997) Drug interactions of clinical significance with selective serotonin reuptake inhibitors. Drug Safety, I7, 390-406.

Movig, K. L. L., Egberts, A. C. G., Lenderink, A.W. et al (2002) Association between antidepressant drugs and hyponatremia: a case-control study. British Journal of Clinical Pharmacology, 53, 363-369.

Muller-Oerlinghausen, B., Berghofer, A. \& Bauer, M. (2002) Bipolar disorder. Lancet, 359, 24I-247.

O'Donovan, C., Hawkes, J. \& Bowen, R. (1993) Effect of lithium dosing schedule on urinary output. Acta Psychiatrica Scandinavica, 87, 92-95.

Stahl, S. M. (1998) Mechanism of action of serotonin selective reuptake inhibitors. Serotonin receptors and pathways mediate therapeutic effects and side effects. Journal of Affective Disorders, 5I, 215-235.

Tatsumi, M., Groshan, K., Blakely, R. D., et al (1997) Pharmacological profile of antidepressants and related compounds at human monoamine transporters. European Journal of Pharmacology, 340, 249-258.

Zullino, D. \& Baumann, P. (200I) Lithium augmentation in depressive patients not responding to selective serotonin reuptake inhibitors. Pharmacopsychiatry, 34, 119-127. 